\title{
Simulating Solar Global Magnetism in 3-D
}

\author{
A. S. Brun and A. Strugarek \\ Laboratoire AIM Paris-Saclay, CEA/Irfu Université Paris-Diderot CNRS/INSU, \\ 91191 Gif-sur-Yvette, France \\ email: sacha.brun@cea.fr
}

\begin{abstract}
We briefly present recent progress using the ASH code to model in 3-D the solar convection, dynamo and its coupling to the deep radiative interior. We show how the presence of a self-consistent tachocline influences greatly the organization of the magnetic field and modifies the thermal structure of the convection zone leading to realistic profiles of the mean flows as deduced by helioseismology.
\end{abstract}

Keywords. Sun, dynamo, tachocline

\section{The Global Sun in 3-D}

Solar magnetism manifests itself on a broad range of scales up to the largest global ones. In this brief report we will summarize the progress we recently made in trying to understand how the Sun generates and maintain its large scale flows and its magnetic field and how these two aspects are linked.

On Figure 1 (left panel) we display the differential rotation realized in our most recent 3-D solar model using the ASH code (Brun et al. 2011). This model couples dynamically for the first time a convective envelope to a deep radiative interior. What is striking in that model is the presence of a tachocline of shear at the base of the convective envelope. Indeed the action of the Coriolis force on the convective motions results in the establishment of a large scale differential rotation. If the Rossby number $(R o=$ $v / 2 \Omega_{0} L$, where $v, L$ are characteristics velocity and length and $\Omega_{0}$ the rotation rate of the star) is less than 1, which is the case for the Sun, Reynolds stresses transport angular momentum equatorward yielding a prograde differential rotation (fast equator, slow poles). The absence of convective motions in the deep interior on the contrary leaves the initial solid body rotation profile unchanged (on the short time scales considered here). A transition between both rotation profile must thus exist, i.e the tachocline. The thermal coupling of both zone further influences the profile of the differential rotation in the convective envelope by tilting the iso-contour of $\Omega$ from cylindrical to conical as inverted by helioseismology. Hence we see that the direct coupling of both zones yield a better description of the inner rotation profile of the Sun. In such coupled models convective overshooting also leads to the excitation of internal waves that can further transport non locally angular momentum thus possibly enforcing solid body rotation but on much longer time scales (Brun et al. 2011).

Such a tachocline will diffuse inward if no processes oppose its slow propagation as was shown by Spiegel \& Zahn in 1992. It has been proposed by Gough \& McIntyre (1998) that a large scale fossil dipolar magnetic field could oppose such spread. In Strugarek et al. 2011a,b, we have studied in 3-D MHD with the ASH code if such a scenario could work. We display on Figure 1 (right panel) the 3 -D rendering of the magnetic field from $\mathrm{r}=0.07$ up to $2.5 \mathrm{R}$. What is directly clear is that the inner fossil field pervades through the convective envelope. This leads to a direct coupling between the differentially rotating 

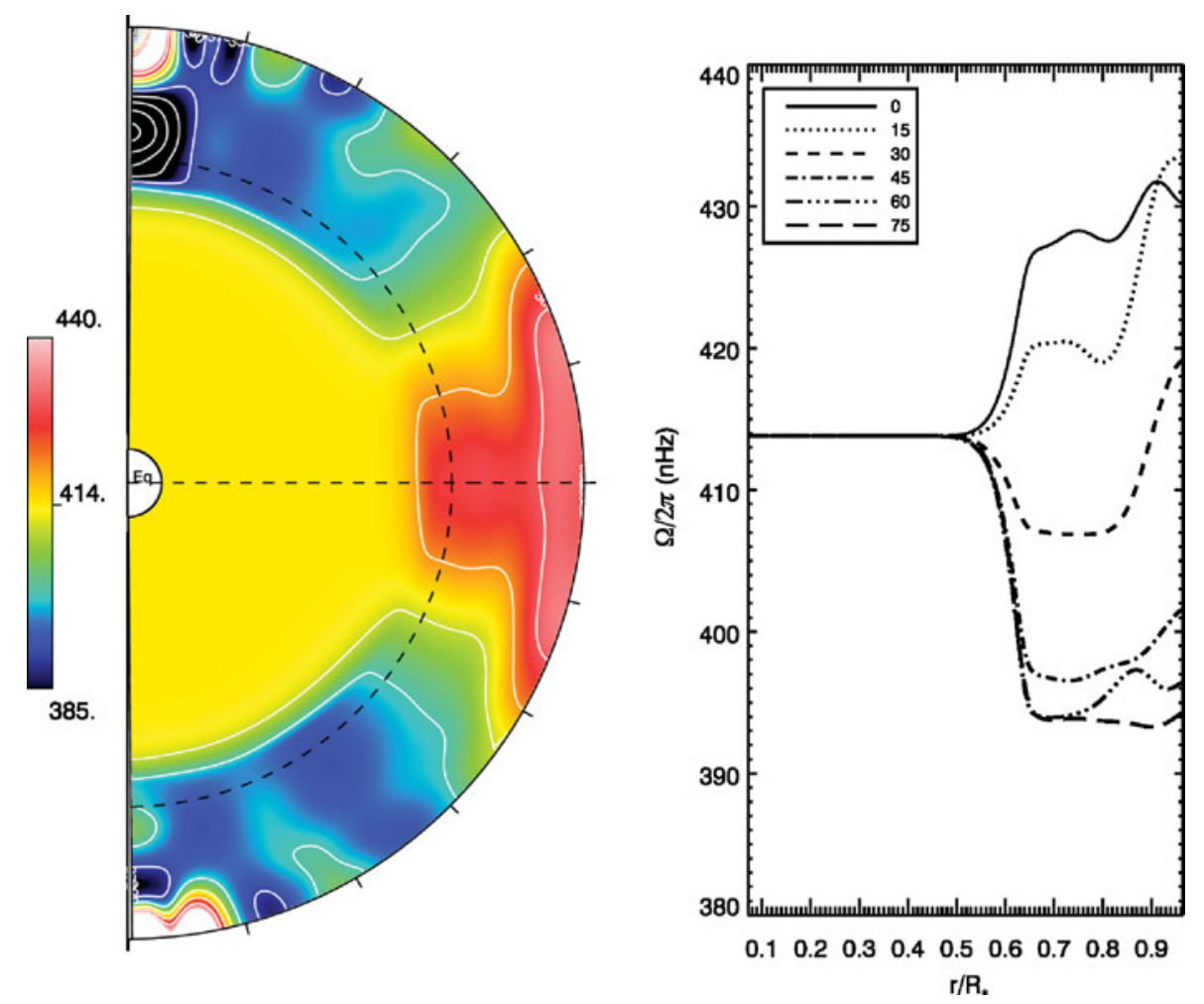

Figure 1. Left: Color contours of the azimuthally and temporally averaged angular velocity realized in a 3-D global solar model coupling the convective envelope to the radiative interior (Brun et al. 2001). Right: 3-D rendering of the magnetic field lines in a global MHD solar model from $r=0.07 R$ up to $r=2.5 R$, using potential field beyond $r=0.97 R$ (Strugarek et al. 2011a).

convective envelope and the deep interior resulting to an efficient angular momentum transport. The end state is an omega profile constant along the poloidal magnetic field (i.e the so called Ferraro's law of iso-rotation) which is not observed. In fact a purely axisymmetric dipolar field is unlikely in the deep interior of the Sun as MHD instabilities exist there and are likely to yield more complex magnetic topologies. In an attempt of taking that into account we have considered non axisymmetric field configurations (oblique and perpendicular dipole), but found that it does not make much differences (Strugarek et al. 2011b). We thus need to consider even more realistic setting or search for different solutions (turbulence, waves) to explain the thinness of the tachocline.

Finally such coupled models are ideal to study the solar global dynamo in particular to understand how the large scale field is being maintained by turbulent flows (Strugarek et al. 2012). Preliminary results show that large scale magnetic field are indeed organized at the base of the convective envelope and in the tachocline confirming the results of Browning et al. (2006). Fast dynamo action is found to operate in the convective envelope with efficient magnetic pumping of field at the base of the convective envelope and in the tachocline, where it is subsequently stretched into large wreaths of horizontal magnetic field. In these new models, a tapering of the diffusivities in the stable zone is present that will hopefully help getting these structures to become buoyant and rise to the surface, much as in the recent work of Nelson et al. (2011) in fastly rotating Suns. 


\section{References}

Browning et al. 2006, ApJ 648, L157

Brun, A. S., Miesch, M. S., \& Toomre 2011, ApJ 742, 79

Gough, D. \& McIntyre, M. 1998, Nature 394, 755

Nelson et al. 2011, ApJ, 739, L38

Strugarek, A., Brun, A. S., \& Zahn, J.-P. 2011a, A\&A 532, 34

Strugarek, A., Brun, A. S., \& Zahn, J.-P. 2011b, AN 332, 891

Strugarek, A., Brun, A. S., Mathis, S., \& Sarazin 2012, ApJ in press

Spiegel, E. A. \& Zahn, J. P. 1992, A\&̈A 265, 106 